\title{
The association between new generation oral contraceptive pill and the development of inflammatory bowel diseases
}

Santosh Sanagapalli ${ }^{1}$, Yanna $\mathrm{Ko}^{1}$, Viraj Kariyawasam ${ }^{1}$, Siew $\mathrm{C} \mathrm{Ng}^{2}$, Whitney Tang ${ }^{2}$, Hithanadura Janaka de Silva $^{3}$, Minhu Chen ${ }^{4}$, Kaichun $\mathrm{Wu}^{5}$, Satimai Aniwan ${ }^{6}$, Ka Kei Ng ${ }^{7}$, David Ong ${ }^{8}$, Qin Ouyang ${ }^{9}$, Ida Hilmi ${ }^{10}$, Marcellus Simadibrata ${ }^{11}$, Pises Pisespongsa ${ }^{12}$, Saranya Gopikrishna ${ }^{1}$, Rupert W Leong ${ }^{1}$, on behalf of the IBD Sydney Organisation and the Asia-Pacific Crohn's and Colitis Epidemiology Study (ACCESS) Group ${ }^{1}$ Gastroenterology and Liver Services, Concord Repatriation General Hospital, Concord NSW, Australia, ${ }^{2}$ Department of Medicine and Therapeutics, State Key Laboratory of Digestive Disease, Institute of Digestive Disease, Li Ka Shing Institute of Health Science, Hong Kong, China, ${ }^{3}$ Faculty of Medicine, University of Kelaniya, Ragama, Sri Lanka, ${ }^{4}$ The First Affiliated Hospital of Sun Yat Sen University, Guangzhou, ${ }^{5}$ Xijing Hospital, Fourth Military Medical University, Xian, China, ${ }^{6}$ King Chulalongkorn Memorial Hospital, Bangkok, Thailand, ${ }^{7}$ Hospital Conde S Januario, Macau, China, ${ }^{8}$ National University Hospital of Singapore, Singapore, ${ }^{9}$ West China Hospital, Sichuan University, Chengdu, China, ${ }^{10}$ University of Malaya Medical Center, Kuala Lumpur, Malaysia, ${ }^{11}$ University of Indonesia, Indonesia, ${ }^{12}$ Maharaj Nakorn Chiangmai Hospital, Chiangmai, Thailand

Background/Aims: To examine the association between use of oral contraceptive pills (OCPs) and the risk of developing inflammatory bowel diseases (IBD), in a modern cohort. Methods: A prospective nested case-control study across sites in the Asia-Pacific region was conducted; involving female IBD cases and asymptomatic controls. Subjects completed a questionnaire addressing questions related to OCP use. Primary outcome was the risk of development of IBD of those exposed to OCP versus non-exposure. Secondary outcomes were development of Crohn's disease (CD) versus ulcerative colitis (UC), and whether age of first use of OCP use may be associated with risk of IBD. Results: Three hundred and forty-eight female IBD cases (41\% $\mathrm{CD}$, median age: 43 years) and 590 female age-matched controls were recruited. No significant association was found between OCP use and the risk of IBD (odds ratio [OR], 1.65; 95\% confidence interval, $0.77-3.13 ; P=0.22)$, CD (OR, 1.55) or UC (OR, 1.01). The lack of association persisted when results were adjusted for age and smoking. IBD cases commenced OCP use at a younger age than controls ( 18 years vs. 20 years, $P=0.049$ ). Conclusions: In this large cohort of subjects from the Asia-Pacific region, we found a modest but not significantly increased risk of developing IBD amongst OCP users. (Intest Res 2018;16:409-415)

Key Words: Inflammatory bowel diseases; Contraceptives, oral

\section{INTRODUCTION}

The pathogenesis of IBD is suspected to be due to genetic and environmental influences. ${ }^{1,2}$ An association between use of the oral contraceptive pill (OCP) with IBD has been

Received November 3, 2017. Revised February 22, 2018.

Accepted February 28, 2018.

Correspondence to Rupert W Leong, Gastroenterology and Liver Services,

Concord Repatriation General Hospital, Concord NSW 2139, Australia. Tel:

+61-2-9767-6111, Fax: +61-2-9767-6767, E-mail: rupertleong@outlook.

com suggested in case control, cohort studies and meta analyses; these studies have predominantly included patient cohorts of European descent. ${ }^{3-6}$ Proposed mechanisms that explain the association include hormonal effects on intestinal permeability, the immune response, promotion of microvascular thrombosis, as well as confounding effects of cigarette smoking. ${ }^{7-12}$

In the decades since their first use, however, the composition of OCPs has transformed dramatically. The newer 3rdand 4th-generation OCPs contain far lower doses of the hormone estrogen, which is thought responsible for many

\footnotetext{
๑ Copyright 2018. Korean Association for the Study of Intestinal Diseases. All rights reserved.

This is an Open Access article distributed under the terms of the Creative Commons Attribution Non-Commercial License (http://creativecommons.org/licenses/by-nc/4.0)

which permits unrestricted non-commercial use, distribution, and reproduction in any medium, provided the original work is properly cited.
} 
of the drug side effects. ${ }^{13-19}$ Similarly, the early generation progestins levonorgestrel and norethisterone were derived from testosterone and subsequently have been replaced by progesterone and spironolactone derivatives; these developments have also contributed to changes in thrombosis risk. ${ }^{13}$ These substantial changes to modern-generation OCPs have modified their biological activities, which may have abrogated their link with the development of IBD. The above mentioned studies that did find a link, all were Western studies that had patient recruitment predominantly prior to the widespread use of modern OCPs ${ }^{3-6}$ Furthermore, some recent clinical studies have found no association between OCP and the development of IBD., ${ }^{2,20-26}$ These studies were, however, limited by small female sample sizes ${ }^{20,23-26}$ or inclusion of males in the total analyses, ${ }^{2,21,25}$ which may result in underpowering and inability to detect an association. Other studies did not evaluate modern OCP formulations. ${ }^{20-22,26}$ None of these studies examined the use of OCP amongst Asian IBD cohorts, whose susceptibility genes differ. ${ }^{27}$

We aimed, therefore, to examine whether the association of IBD with OCP exists in a contemporary cohort of patients using modern formulations of OCP. In addition, we proposed to examine for the presence of this association amongst an Asia-Pacific cohort for the first time.

\section{METHODS}

\section{Study Design}

This prospective nested case-control study recruited consecutive patients across sites in the Asia-Pacific region recruited from 2011. The primary outcome was the risk of development of IBD of those exposed to OCP prior to the development of IBD versus non-exposure. Secondary outcome measures were development of $\mathrm{CD}$ versus $\mathrm{UC}$, and whether duration of OCP use may be associated with the risk of IBD.

\section{Study Population}

Ambulatory cases and controls were prospectively collected between 2011 and 2013 from the Asia-Pacific region from the Asia-Pacific Crohn's and Colitis Epidemiology Study (ACCESS) cohort ${ }^{28-30}$ and the Sydney IBD Cohort. ${ }^{31,32}$ Countries involved were Australia, China, Hong Kong, Indonesia, Macau, Malaysia, Singapore, Sri Lanka and Thailand. Cases comprised of adult female IBD cases with confirmed diagnosis by a gastroenterologist based on clinical features, endoscopic findings, histology, radiology and response to treatment. The Australian cohort included incident and prevalent IBD cases whereas the Asian cohort included only incident cases. Exclusion criteria were male sex, first OCP use after IBD diagnosis, inconclusive diagnosis of IBD or those with a diagnosis of IBD-unclassified. These diagnostic criteria as well as patient demographics were standardized across all study sites. Controls were age-matched asymptomatic subjects without a family history of IBD, randomly recruited from the streets or department stores within the same communities as the cases. All participants had equal access to healthcare. Family members of IBD cases were not included as controls.

\section{Questionnaire}

Cases and controls were administered a detailed questionnaire that explored exposure to a number of established and postulated environmental risk factors for IBD, including questions related to OCP use. In Asia, the questionnaire from the international organization of IBD regarding environmental factors was used; it has been used previously in a number of other IBD cohorts. ${ }^{33-35}$ In Australia, ethics committee approval had already been obtained to utilize the validated New Zealand questionnaire of Gearry et al. ${ }^{21}$ Both questionnaires covered use of OCP in detail and as such, data could be combined. Questions covered included the history of any lifetime exposure to OCP and date of first use. However, individually they were not powered to observe these differences. ${ }^{2,28}$ At least 1 month of OCP use was required to define a subject as exposed to OCP. Smoking status was ascertained. Participants were encouraged to take time to complete the questionnaire and to consult with family members to improve its accuracy. Categorical options of "yes," "no" and "unsure" were given to the participants where possible to reduce the risk of recall bias.

\section{Sample Size Calculation}

Previous studies finding an association between OCP use and IBD have reported ORs ranging between 1.5 and $2.8{ }^{3}$ To detect an OR of at least 1.5 using a two-tailed test with $80 \%$ power and an alpha level of $5 \%$, assuming exposure to OCPs in the controls of $30 \%$ and a control to case ratio of 3 to 2, a sample size of at least 348 cases and 522 controls was required. As recruitment proceeded, it became evident that the OCP exposure rate amongst controls was only $27 \%$. We therefore increased subject recruitment to account for the OCP exposure. 


\section{Statistical Analysis}

Descriptive nonparametric continuous variables of age and duration of IBD were expressed as medians and interquartile ranges (IQR) and compared statistically using the Mann Whitney $U$-test. Categorical variables were described as counts and percentages and compared with the chisquare test. OR for developing IBD according to exposure to OCPs by univariate analysis with $95 \%$ CI were calculated, as were the same for the risk of CD and UC. A multivariate model was then calculated using logistic regression to adjust for the possible confounders of smoking status and age, as well as Asian versus Australian cohort. A P-value of $<0.05$ was considered statistically significant. Statistical analysis was performed using SPSS for Mac version 20.0 (IBM Corp., Armonk, NY, USA).

\section{Ethical Considerations}

The local Ethics Committees of each center approved the study and written informed consent was obtained from each subject.

\section{RESULTS}

In total, 348 IBD female cases and 590 female controls fulfilled the criteria and were included in the study. Of these, 142 (40.8\%) had CD and 206 (59.2\%) had UC. Median age at recruitment was 43.0 years in IBD cases (IQR, 31.0-55.0 years) and 42.0 years in controls (IQR, 28.0-56.0 years) $(P=0.42)$. Amongst OCP users, the median age of first use was slightly younger in IBD cases, at 18.0 years (IQR, 14.521.5 years); this compared with 20.0 years (IQR, 16.5-23.5 years; $P=0.049$ ). There was no significant difference in the median age and prevalence of smoking between cases and controls. Table 1 demonstrates these demographic characteristics of the participants.

Of those with IBD, $31 \%$ had been exposed to OCP $(28 \%$ exposed amongst UC cases, 35\% exposed amongst CD patients). This compares to an OCP exposure rate of $27 \%$ amongst the controls. The OCP exposure rate amongst controls was lower in the Asian compared to the Australian cohort ( $24 \%$ vs. $34 \% ; P<0.01)$. The median year of first use of OCP amongst the cohort was 1997 (IQR, 1992-2005). The median duration of disease was 11.0 years in the Australian cohort (IQR, 6.6-15.4 years), and 5.0 years in the Asian cohort (IQR, 4.0-6.0 years).

There was no significant association between OCP use and risk of developing IBD. Compared with women who had never used OCPs, the ORs for IBD for those who had been exposed to OCPs was 1.65 (95\% CI, 0.77-3.13; $P=0.22$ ). The ORs for CD and UC amongst those who had been exposed to OCPs were similarly nonsignificant, at $1.55(P=0.22)$ and $1.01(P=0.96)$ respectively. Table 2 describes the unadjusted risks for the development of IBD, CD and UC with OCP use.

We considered the possibility that the relative contraindication of OCPs in women who smoke may have had some influence on our results. We also considered that differences between the Asian and Australian cohorts might have had an impact. Hence, we performed a multivariate analysis adjusting for smoking status, age, as well as Asian versus Australian cohort type (see Table 3). On multivariate analysis, the OR for IBD amongst those who were exposed to OCP was 1.35, and remained nonsignificant (95\% CI, 0.60-2.10, $P=0.44$ ). Adjusted ORs for CD and UC also remained nonsignificant, at $1.31(P=0.32)$ and $1.20(P=0.47)$ respectively. Further sub classification of ethnicity amongst the Australian (European vs. Middle Eastern ethnic origin) and Asian cohorts (Chinese

Table 1. Characteristics of Study Participants

\begin{tabular}{lccc}
\hline \multicolumn{1}{c}{ Variable } & Cases & Control & $P$-value \\
\hline Total no. & 348 & 590 & \\
CD & $142(40.8)$ & - & \\
UC & $206(59.2)$ & - & \\
Age (yr) & $43(31-55)$ & $42(28-56)$ & 0.42 \\
Smoking ever & $72(20.7)$ & $116(19.7)$ & 0.65 \\
\hline Values are presented as number (\%) or median (interquartile range).
\end{tabular}

Table 2. Unadjusted ORs for IBD with OCP Use

\begin{tabular}{lccc}
\hline Risk with OCP use & OR & $\mathbf{9 5 \%}$ Cl & $P$-value \\
\hline IBD & 1.65 & $0.77-3.13$ & 0.22 \\
CD & 1.55 & $0.78-3.10$ & 0.22 \\
UC & 1.01 & $0.62-1.66$ & 0.96 \\
\hline
\end{tabular}

$\mathrm{OCP}$, oral contraceptive pill.

Table 3. ORs for IBD with OCP Use, Adjusted for Age, Smoking Status, and Asian versus Australian Cohort

\begin{tabular}{lccc}
\hline Risk with OCP use & OR & $\mathbf{9 5 \% ~ C l}$ & $\boldsymbol{P}$-value \\
\hline IBD & 1.35 & $0.60-2.10$ & 0.44 \\
CD & 1.31 & $0.55-1.99$ & 0.32 \\
UC & 1.20 & $0.70-1.70$ & 0.47 \\
\hline
\end{tabular}

$\mathrm{OCP}$, oral contraceptive pill. 
vs. non-Chinese) did not reveal any statistically significant differences (all $P>0.05$ ); though, the study sample size was unlikely to be sufficiently powered to demonstrate differences.

\section{DISCUSSION}

A large cohort study ${ }^{5}$ and meta analyses ${ }^{3,6}$ linked the association between OCP use and IBD. This link has been confirmed in other observational and case control studies. ${ }^{7,36,37}$ However the vast majority of patients included in these studies were recruited in an era when newer generation OCPs were not yet introduced or widely used, including some from the 1960s, when the first generation OCPs were used. The composition of these OCPs bear little resemblance to the formulations used today. ${ }^{38}$ Even trials identified by the meta-analysis as examining low dose OCPs due to more recent publication in fact still had patient recruitment predominantly prior to $1990 .^{36,39,40}$ Modern OCPs, in contrast to OCPs used in publications that found their association with IBD, were designed to have a minor impact on metabolic and vascular risk factors. The biological effects of newer generations of OCP, therefore, may no longer be relevant to the development of IBD.

Some case control studies on the environmental risk factors associated did not find an association between OCP use with IBD. ${ }^{2,20-26}$ However, these studies were not specifically designed to study the relationship between OCPs and IBD. They suffered from limitations, such as inclusion of males in the analysis, ${ }^{2,21,25}$ insufficient number of female study participants, ${ }^{20,23-26}$ low rates of OCP use, ${ }^{25}$ and still being conducted too long ago to account for changing patterns of OCP use. .0-22,26 $^{2}$

On the other hand, the present study was not hindered by any of these limitations. We examined a more modern cohort with the majority of OCP users having done so after 1995, a period when third- and fourth-generation OCPs have become the dominant type used in a number of countries worldwide. ${ }^{41,42}$ In this cohort, we found a very modest association between OCP use and the risk of developing IBD (OR=1.65, $P=0.22)$, in particular CD (OR=1.55, $P=0.22)$; though this association did not reach statistical significance. In fact, the ORs we observed were not far lower in magnitude than some previous studies that described statistically significant associations. ${ }^{3}$ The implication is that the association between OCP use and IBD may remain even in the era of modern-generation oral contraceptives, but this could only be determined with certainty by an even more highly powered study. Our findings therefore reiterate the need for more up to date studies to validate such an association that has been purported based on observational studies with such modest ORs in the past.

An important strength of the study is related to its evaluation of the OCP association in an Asia-Pacific setting. While previous studies reporting on IBD risk relating to OCP use have focused primarily on Western populations, this is the first study examining the association among an Asian population. Cases were prospectively included and followed, thereby overcoming the issues with bias associated with subjects from referral centers. Cases and controls were well matched and drawn from the same geography and population setting, thus being representative of the general population of these areas. ${ }^{28}$

A limitation of this study is its questionnaire-based style, with the associated risks of recall bias. However, the decision to use an OCP is a significant one in most women's lives, is often used long term, and often involves discussion with family members and friends; therefore it would be less prone to such bias. Another limitation of the study is that, whilst unlike previous large studies it was amongst women predominantly taking OCP in the era of newer formulation, we were unable to obtain precise data regarding specific brand of OCP used; hence, it is inevitable that some had used older formulations. It is likely, however, that family physicians would prescribe the safer newer generation OCPs rather than older formulations that are more prone to adverse effects. Similarly, we also acknowledge that we did not have access to data regarding dosage and precise duration of OCP use, other than the fact that subjects had used the drug for at least 1 month; therefore we cannot confirm or exclude the possibility that these factors may have an impact on the results.

While we identified differences between the Australian and Asian cohorts in our study, no significant association with IBD was found when controlling for this. Our findings suggest that OCP use, as a risk factor, may be equally applicable in Asian IBD as it is in Western. This is despite the well-described and clinically important differences in susceptibility genes found in Asian IBD, ${ }^{27}$ and contrasts with the observations that other environmental risk factors such as smoking and childhood antibiotic use may play less of a role, or even an inverse role, in Asian IBD compared to Western. ${ }^{28}$ The findings of the present study contribute to our understanding of our pathogenesis of IBD; could OCP use be one of the "Westernization" factors contributing to the rapidly rising incidence of IBD in Asia and other traditionally lowincidence regions? 
In conclusion, we demonstrated a modest though nonsignificant association, not dissimilar to that described in previous studies, between the use of OCPs and the development of IBD in this large multicenter case control study; the first of its kind in the Asia-Pacific region and to study women in the era of modern-generation oral contraceptives. OCP use was not associated with a significantly different risk of IBD in an Asian cohort compared to a Western cohort.

\section{FINANCIAL SUPPORT}

This study was funded in part by an unrestricted educational grant from Ferring Pharmaceuticals, Hong Kong; and Direct Grant Faculty of Medicine Chinese University of Hong Kong. These grants were used to support the database setup and maintenance of the study. The study sponsors had no contribution in the study design, analysis, interpretation of data or publication.

\section{CONFLICT OF INTEREST}

No potential conflict of interest relevant to this article was reported.

\section{AUTHOR CONTRIBUTION}

Guarantor of article: Santosh Sanagapalli.

Specific author contributions: S.S. conceived the study, analyzed the data and wrote the manuscript. Y.K. performed statistical analyses, acquired data and contributed to manuscript review. S.C.N. treated the studied patients, acquired data and contributed to critical review of the manuscript. W.T. performed data entry, data analysis and study audit. V.K., H.J.S., M.C., K.W., S.A., K.K.N., D.O., Q.O., I.H., M.S. and P.P. contributed to treatment of studied patients, data acquisition and manuscript review. S.G. performed analysis and interpretation of data and contributed to review of the manuscript. R.W.L. also conceived the study and critically reviewed the manuscript. All authors have seen and approved the final manuscript.

\section{ACKNOWLEDGEMENTS}

The authors are thankful to Dr. Anuradhani Kasturiratne, Dr. Yasith Udara Senanayake (Sri Lanka), Prof. PinJin Hu, Dr. Zhenhua Zhu, Prof. Zhirong Zeng (Guangzhou), Dr. Xin Wang (Xian), Prof. Sathaporn Manatsathit (Bangkok), Dr. Hon Ho Yu, Dr. Mo Fong Li (Macau), Dr. Choon Jin Ooi, Dr.
Khoon-Lin Ling (Singapore), Dr. Yu-Fang Wang (Chengdu), Prof. Khean Lee Goh (Malaysia), Dr. Murdani Abdullah (Indonesia) and all other gastroenterologists and staff at every hospital who have assisted in identification and recruitment of patients.

\section{REFERENCES}

1. Abraham C, Cho JH. Inflammatory bowel disease. N Engl J Med 2009;361:2066-2078.

2. Ko Y, Kariyawasam V, Karnib M, et al. Inflammatory bowel disease environmental risk factors: a population-based casecontrol study of Middle Eastern Migration to Australia. Clin Gastroenterol Hepatol 2015;13:1453-1463.e1.

3. Cornish JA, Tan E, Simillis C, Clark SK, Teare J, Tekkis PP. The risk of oral contraceptives in the etiology of inflammatory bowel disease: a meta-analysis. Am J Gastroenterol 2008;103:23942400

4. Godet PG, May GR, Sutherland LR. Meta-analysis of the role of oral contraceptive agents in inflammatory bowel disease. Gut 1995;37:668-673.

5. Khalili H, Higuchi LM, Ananthakrishnan AN, et al. Oral contraceptives, reproductive factors and risk of inflammatory bowel disease. Gut 2013;62:1153-1159.

6. Ortizo R, Lee SY, Nguyen ET, Jamal MM, Bechtold MM, Nguyen DL. Exposure to oral contraceptives increases the risk for development of inflammatory bowel disease: a meta-analysis of case-controlled and cohort studies. Eur J Gastroenterol Hepatol 2017;29:1064-1070.

7. Corrao G, Tragnone A, Caprilli R, et al. Risk of inflammatory bowel disease attributable to smoking, oral contraception and breastfeeding in Italy: a nationwide case-control study. Cooperative Investigators of the Italian Group for the Study of the Colon and the Rectum (GISC). Int J Epidemiol 1998;27:397-404.

8. Khalili H. Risk of inflammatory bowel disease with oral contraceptives and menopausal hormone therapy: current evidence and future directions. Drug Saf 2016;39:193-197.

9. Wakefield AJ, Sawyerr AM, Hudson M, Dhillon AP, Pounder RE Smoking, the oral contraceptive pill, and Crohn's disease. Dig Dis Sci 1991;36:1147-1150.

10. Looijer-van Langen M, Hotte N, Dieleman LA, Albert E, Mulder C, Madsen KL. Estrogen receptor-beta signaling modulates epithelial barrier function. Am J Physiol Gastrointest Liver Physiol 2011;300:G621-G626. doi: 10.1152/ajpgi.00274.2010.

11. Bouman A, Heineman MJ, Faas MM. Sex hormones and the immune response in humans. Hum Reprod Update 2005;11:411423. 
12. Cutolo M, Sulli A, Straub RH. Estrogen metabolism and autoimmunity. Autoimmun Rev 2012;11:A460-A464.

13. Stewart M, Black K. Choosing a combined oral contraceptive pill. Aust Prescr 2015;38:6-11.

14. Parkin L, Sharples K, Hernandez RK, Jick SS. Risk of venous thromboembolism in users of oral contraceptives containing drospirenone or levonorgestrel: nested case-control study based on UK General Practice Research Database. BMJ 2011;342:d2139. doi: 10.1136/bmj.d2139.

15. Jick SS, Hernandez RK. Risk of non-fatal venous thromboembolism in women using oral contraceptives containing drospirenone compared with women using oral contraceptives containing levonorgestrel: case-control study using United States claims data. BMJ 2011;342:d2151. doi: 10.1136/bmj.d2151.

16. Seeger JD, Loughlin J, Eng PM, Clifford CR, Cutone J, Walker AM. Risk of thromboembolism in women taking ethinylestradi$\mathrm{ol} /$ drospirenone and other oral contraceptives. Obstet Gynecol 2007;110:587-593.

17. van Hylckama Vlieg A, Helmerhorst FM, Vandenbroucke JP, Doggen CJ, Rosendaal FR. The venous thrombotic risk of oral contraceptives, effects of oestrogen dose and progestogen type: results of the MEGA case-control study. BMJ 2009;339:b2921. doi: 10.1136/bmj.b2921.

18. Dinger JC, Heinemann LA, Kühl-Habich D. The safety of a drospirenone-containing oral contraceptive: final results from the European Active Surveillance Study on oral contraceptives based on 142,475 women-years of observation. Contraception 2007;75:344-354.

19. Baillargeon JP, McClish DK, Essah PA, Nestler JE. Association between the current use of low-dose oral contraceptives and cardiovascular arterial disease: a meta-analysis. J Clin Endocrinol Metab 2005;90:3863-3870.

20. Han DY, Fraser AG, Dryland P, Ferguson LR. Environmental factors in the development of chronic inflammation: a casecontrol study on risk factors for Crohn's disease within New Zealand. Mutat Res 2010;690:116-122.

21. Gearry RB, Richardson AK, Frampton CM, Dodgshun AJ, Barclay ML. Population-based cases control study of inflammatory bowel disease risk factors. J Gastroenterol Hepatol 2010;25:325333.

22. Bernstein CN, Rawsthorne P, Cheang M, Blanchard JF. A population-based case control study of potential risk factors for IBD. Am J Gastroenterol 2006;101:993-1002.

23. Vcev A, Pezerovic D, Jovanovic Z, Nakic D, Vcev I, Majnarić L. A retrospective, case-control study on traditional environmental risk factors in inflammatory bowel disease in Vukovar-Srijem County, north-eastern Croatia, 2010. Wien Klin Wochenschr 2015;127:345-354
24. Hansen TS, Jess T, Vind I, et al. Environmental factors in inflammatory bowel disease: a case-control study based on a Danish inception cohort. J Crohns Colitis 2011;5:577-584.

25. Jiang L, Xia B, Li J, et al. Risk factors for ulcerative colitis in a Chinese population: an age-matched and sex-matched casecontrol study. J Clin Gastroenterol 2007;41:280-284.

26. Sicilia B, Arribas F, Nerín J, López Miguel C, Vicente R, Gomollón F. Risk factors for ulcerative colitis: a population-based, case-control study in Spain. J Crohns Colitis 2008;2:158-161.

27. Ng SC, Tsoi KK, Kamm MA, et al. Genetics of inflammatory bowel disease in Asia: systematic review and meta-analysis. Inflamm Bowel Dis 2012;18:1164-1176.

28. Ng SC, Tang W, Leong RW, et al. Environmental risk factors in inflammatory bowel disease: a population-based case-control study in Asia-Pacific. Gut 2015;64:1063-1071.

29. Ng SC, Zeng Z, Niewiadomski O, et al. Early course of inflammatory bowel disease in a Population-Based Inception Cohort Study from 8 countries in Asia and Australia. Gastroenterology 2016;150:86-95.e3.

30. Ng SC, Tang W, Ching JY, et al. Incidence and phenotype of inflammatory bowel disease based on results from the AsiaPacific Crohn's and colitis epidemiology study. Gastroenterology 2013;145:158-165.e2.

31. Selinger CP, Andrews JM, Titman A, et al. Long-term follow-up reveals low incidence of colorectal cancer, but frequent need for resection, among Australian patients with inflammatory bowel disease. Clin Gastroenterol Hepatol 2014;12:644-650.

32. Selinger CP, Andrews J, Dent OF, et al. Cause-specific mortality and 30-year relative survival of Crohn's disease and ulcerative colitis. Inflamm Bowel Dis 2013;19:1880-1888.

33. Halfvarson J, Jess T, Magnuson A, et al. Environmental factors in inflammatory bowel disease: a co-twin control study of a Swedish-Danish twin population. Inflamm Bowel Dis 2006;12:925933.

34. Jakobsen C, Paerregaard A, Munkholm P, Wewer V. Environmental factors and risk of developing paediatric inflammatory bowel disease: a population based study 2007-2009. J Crohns Colitis 2013;7:79-88.

35. Vind I, Riis L, Jespersgaard C, et al. Genetic and environmental factors as predictors of disease severity and extent at time of diagnosis in an inception cohort of inflammatory bowel disease, Copenhagen County and City 2003-2005. J Crohns Colitis 2008;2:162-169

36. García Rodríguez LA, González-Pérez A, Johansson S, Wallander MA. Risk factors for inflammatory bowel disease in the general population. Aliment Pharmacol Ther 2005;22:309-315. 
37. Sandler RS, Wurzelmann JI, Lyles CM. Oral contraceptive use and the risk of inflammatory bowel disease. Epidemiology 1992;3:374-378.

38. Christin-Maitre S. History of oral contraceptive drugs and their use worldwide. Best Pract Res Clin Endocrinol Metab 2013;27:3-12.

39. Card T, Logan RF, Rodrigues LC, Wheeler JG. Antibiotic use and the development of Crohn's disease. Gut 2004;53:246-250.
40. Sicilia B, López Miguel C, Arribas F, López Zaborras J, Sierra E, Gomollón F. Environmental risk factors and Crohn's disease: a population-based, case-control study in Spain. Dig Liver Dis 2001;33:762-767.

41. Lewis MA, MacRae KD, Kühl-Habichl D, Bruppacher R, Heinemann LA, Spitzer WO. The differential risk of oral contraceptives: the impact of full exposure history. Hum Reprod 1999;14:1493-1499.

42. Wilson NM, Laursen M, Lidegaard Ø. Oral contraception in Denmark 1998-2010. Acta Obstet Gynecol Scand 2012;91:810815. 